\title{
Patient preferences for anaesthesia management during cataract surgery
}

\author{
D S Friedman, S W Reeves, E B Bass, L H Lubomski, L A Fleisher, O D Schein
}

Br J Ophthalmol 2004;88:333-335. doi: 10.1136/bjo.2003.028258

\begin{abstract}
Background/aims: To assess patient preferences for different anaesthesia management strategies during cataract surgery.

Methods: Cross sectional clinic based study of patient preferences for anaesthesia management strategies. Patients rated their preferences using a linear rating scale from 0 to 100.

Results: Subjects tended to prefer block to topical anaesthesia and oral to intravenous sedation. On a scale from 0 to 100 , subjects preferred oral to intravenous sedation and block to topical anaesthesia by about 8 points.

Conclusions: When given the choice of four different anaesthesia management strategies, $72 \%$ of the study subjects preferred block anaesthesia to topical anaesthesia. More patients chose to have oral sedation than intravenous sedation. These findings indicate that patients may prefer anaesthesia management approaches other than the ones they are currently being offered.
\end{abstract}

$\mathrm{T}$ he use of topical anaesthesia for cataract surgery has increased dramatically in the past 5 years. Data from the Study of Medical Testing for Cataract Surgery $\left(\right.$ SMTCS $^{1}$ and a recent review of the literature ${ }^{2}$ conclude that while topical and block anaesthesia are both highly successful, patients report slightly greater pain with topical anaesthesia .

Patient preferences for different sedation strategies are poorly understood. Sedation currently ranges from no sedation whatsoever to heavy sedation mimicking general anaesthesia. Higher intensity sedation requires more interventions to control the heart rate and rhythm and increases the likelihood of oxygen desaturation during surgery. ${ }^{1}$ While these adverse events rarely lead to more serious outcomes, patients may be willing to sacrifice some degree of sedation to avoid them. The present study is an attempt to understand the patient perspective on anaesthesia care during cataract surgery, focusing on patient preferences for topical and block local anaesthesia and higher and lower intensity sedation strategies.

\section{METHODS}

\section{Study population}

Volunteers were either individuals presenting for routine eye evaluation at the Wilmer Eye Institute or accompanying friends or spouses, who were all over the age of 40 years. All interviews were conducted from March to June 2000. All subjects gave verbal informed consent to the interview in accordance with the Johns Hopkins institutional review board.

Patients were presented with scenarios describing the anaesthesia experience. These were designed using published data to estimate the likelihood of pain during the adminis-

tration of the block and during surgery as well as the side effects using different approaches to ocular anaesthesia and sedation. ${ }^{1-3}$ The scenarios were written at a seventh grade reading level and props summarising the scenarios were employed as aids (fig l).

We did not think that patients could put the brief anaesthesia experience on a life and death scale using either a standard gamble ${ }^{4}$ or time trade-off technique. ${ }^{5}$ Instead, subjects were asked to choose which of two approaches was preferred, and to rate the two approaches on a scale from 0 to 100 with 0 being the worst possible anaesthesia experience and 100 being a perfect experience.

Scenarios described four of the most commonly used approaches to anaesthesia management for cataract surgery: (1) oral sedation with topical drops; (2) oral sedation with block anaesthesia; (3) intravenous sedation with topical drops; and (4) intravenous sedation with block anaesthesia. Subjects were presented two options for anaesthesia management and asked to choose one as preferred. A visual aid was used to allow the patient to rate the described techniques on a scale from 0 to 100 with the anchor "worst experience" at 0 and the anchor "perfect experience" at 100. Before the interview we described the "perfect experience" in the following way: "(1) You feel perfectly calm before and during the surgery; (2) you feel absolutely no pain or discomfort at any point during the operation; (3) there is no risk of complications during the operation; (4) you feel perfectly well at the end of the operation; and (5) you can see out of your eye immediately and go home as soon as you like." We described the "worst experience" in the following way: "(1) You feel very anxious before and during the operation; (2) you have lots of pain and discomfort during surgery; (3) there is a large risk of complications during surgery; (4) you feel terrible at the end of the operation; and (5) you cannot see out of your eye for many hours after surgery, and have to wait several hours in the hospital before you can go home."

\section{Option 1}

- Valium pill before surgery relaxes you during surgery

- Injection for numbing

- Moderate pain during injection

- No sight during surgery

- 1/30 people feel some mild pain during surgery

- Patch worn for 6 hours after surgery, then sight returns

Figure 1 Prop used for clarifying scenarios to subjects. 


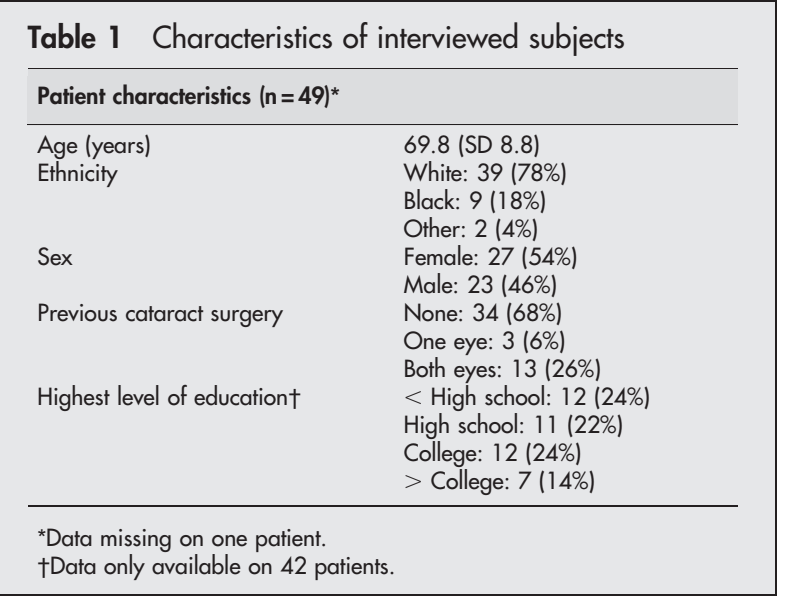

For each scenario we estimated the risk of adverse events using the published literature as follows.

\section{Block versus topical anaesthesia}

For the block with oral sedation strategy we stated that the subject would "feel moderate pain for 10-20 seconds during the injection, like when the dentist numbs your teeth." The estimated incidence for pain being felt at all during surgery with block anaesthesia was 3\% versus $10 \%$ with topical. ${ }^{1}$ For topical anaesthesia we estimated subjects would probably see movements and colours, and that $25 \%$ of the time subjects would see the instruments or the surgeon's hands. ${ }^{3}$ Finally, we informed the subjects that with block anaesthesia they would not be able to see out of the operated eye for 6 hours, while with topical anaesthesia recovery would occur within 15 minutes.

\section{Oral versus intravenous sedation}

Based on the results of the Study of Medical Testing for Cataract Surgery (SMTCS) we estimated that for patients receiving oral sedation alone nausea or vomiting would be a rare event, while for those receiving intravenous sedation, 3\% would experience this adverse event. Additionally, $1 \%$ of those receiving oral sedation would require medical treatment intraoperatively to control high blood pressure or a slow heart rate as opposed to $3 \%$ of those receiving intravenous sedation. Finally, we told patients that "most of the time" that they would feel drowsy for several hours after the surgery with oral sedation, while they would be drowsy only in $10 \%$ of cases after receiving intravenous sedation.

\section{RESULTS}

Fifty subjects consented to the interview. The average age of subjects was 69.8 years, $78 \%$ were white, and 54\% were female (table 1).

Subjects tended to prefer block to topical anaesthesia and oral to intravenous sedation in most side by side comparisons (table 2). In general, about two thirds of individuals preferred oral to intravenous sedation, and a similar proportion favoured block over topical anaesthesia $(p<0.05$ for both comparisons). On a scale from 0 to 100 , patients preferred oral to intravenous sedation and block to topical by about eight points. However, individuals who had previously had cataract surgery preferred topical and block anaesthesia equally $(56 \%$ preferred topical when using oral sedation, while 50\% preferred topical anaesthesia with intravenous sedation, $\mathrm{p}>0.2$ ). Those with previous surgery preferred oral to intravenous sedation at similar rates to the rest of the group.

When asked to state the preferred strategy after rating these side by side comparisons, subjects preferred intravenous sedation plus block and the oral sedation plus block strategies in equal proportions (36\% each), with an additional $26 \%$ preferring oral sedation plus topical anaesthesia.

\section{DISCUSSION}

This is the first study to question patients directly regarding their preferences for different anaesthesia management strategies that are currently used for cataract surgery. Patients tended to prefer block anaesthesia to topical anaesthesia when given an explanation of the known risks and benefits of treatment. Furthermore, oral sedation was generally preferred to intravenous sedation. When asked to choose one approach, patients preferred block to topical anaesthesia, with $72 \%$ choosing block anaesthesia. However, those who had previously had cataract surgery chose topical and block anaesthesia in equal proportions.

Our estimates of adverse events related to different sedation strategies were largely based on the results of a study that collected detailed data on close to 20000 cataract procedures from a wide range of practices in the United States and Canada. ${ }^{1}$ The estimates of the likelihood of these outcomes is therefore representative, but individual practitioners may have different rates of adverse events. For example, faster surgeons may find that their patients have lower rates of sedation related adverse events. Our recent review of the literature indicates that retrobulbar, peribulbar, and sub-Tenon's blocks provide similar pain control for patients. ${ }^{2}$

We emphasised that the cataract operation would be successful regardless of the anaesthesia approach chosen and highlighted for patients the differences between the two

Table 2 Responses to scenarios

\begin{tabular}{|c|c|c|}
\hline Comparisons & Proportion selecting & $\begin{array}{l}\text { Rating from 1-100 } \\
\text { Mean (SD) }\end{array}$ \\
\hline \multicolumn{3}{|c|}{ Oral sedation+block anaesthesia $v$ oral sedation+topical anaesthesia } \\
\hline Oral+block & $66 \%$ & $63.7(22.1)$ \\
\hline Oral+topical & $34 \%$ & $55.8(25.0)$ \\
\hline \multicolumn{3}{|c|}{ IV sedation+block anaesthesia v IV sedation+topical anaesthesia } \\
\hline IV+block & $68 \%$ & $70.2(20.0)$ \\
\hline IV+topical & $32 \%$ & $62.8(18.2)$ \\
\hline \multicolumn{3}{|c|}{ IV sedation+block anaesthesia $v$ oral sedation+block anaesthesia } \\
\hline IV+block & $34 \%$ & $59.6(22.9)$ \\
\hline Oral+block & $66 \%$ & $66.2(19.5)$ \\
\hline \multicolumn{3}{|c|}{ IV sedation+topical anaesthesia $v$ oral sedation+topical anaesthesia } \\
\hline IV+topical & $34 \%$ & $59.3(19.4)$ \\
\hline Oral+topical & $66 \%$ & $67.8(17.22)$ \\
\hline
\end{tabular}


sedation or block scenarios with a card showing the expected rates of events in a side by side comparison. We did not coach patients or try to redirect them when responses were inconsistent. Finally, a single trained individual interviewed all patients in a standardised fashion.

Our study is limited by the difficulties inherent in describing the anaesthesia experience both succinctly and completely. In pilot testing we found that giving too much information was overwhelming for patients. We therefore changed one variable at a time in order to assess different sedation and local anaesthesia approaches separately. This aided patient comprehension significantly, but may have limited how completely the subjects understood the anaesthesia experience. In addition, how questions are phrased can influence the responses that patients give. We avoided biasing statements and limited our explanations to the key differences associated with different approaches. However, different investigators phrasing the scenarios with alternative wording may have obtained different results. Finally, the patient population studied may have affected the responses obtained. For example, there were significantly different responses regarding topical versus block anaesthesia depending on whether or not patients had previously undergone cataract surgery. We attempted to recruit a wide range of individuals representative of clinical practice by recruiting patients from different sites within our institution. The sex, racial, and educational variation among the respondents indicates that we sampled a relatively diverse population.

In conclusion, we found that $72 \%$ of subjects preferred block anaesthesia to topical anaesthesia, but that patients with previous surgery chose topical anaesthesia about half the time. More patients chose to have oral sedation than intravenous sedation. These findings indicate that patients may or may not want the anaesthesia approach that surgeons are choosing for them. More research is required to confirm these findings and to probe the aetiology of these different preferences. Patients may be more fearful of pain if they have not undergone cataract surgery, leading them to choose block anaesthesia over topical. Comparative studies of different sedation strategies focused on patient reported outcomes would greatly help in determining the optimal management strategy for anaesthesia care during cataract surgery.

\section{ACKNOWLEDGEMENTS}

DSF is funded in part by NEI grant K23-EY00358 and is the Robert E McGinn Scholar from Research to Prevent Blindness; ODS is funded in part by NEI grant K24-EY00395 and the Burton E Grossman Program for Preventive Ophthalmology.

\section{Authors' affiliations}

D S Friedman, L H Lubomski, O D Schein, Department of Ophthalmology, Johns Hopkins University, Baltimore, MD, USA

S W Reeves, School of Medicine, Johns Hopkins University, Baltimore, $M D$, USA

Eric B Bass, Department of Medicine, Department of Health Policy and Management, Johns Hopkins University, Baltimore, MD, USA

L A Fleisher, Department of Anesthesiology, Johns Hopkins University, Baltimore, MD, USA

Correspondence to: David S Friedman, MD, MPH, 120 Wilmer Building, Johns Hopkins Hospital, $600 \mathrm{~N}$ Wolfe Street, Baltimore, MD 21287-9019, USA; david.friedman@jhu.edu

Accepted for publication 2 July 2003

\section{REFERENCES}

1 Katz J, Feldman MA, Bass EB, et al. Injectable versus topical anesthesia for cataract surgery: patient perceptions of pain and side effects. Ophthalmology 2000; 107:2054-60.

2 Friedman DS, Bass EB, Lubomski LL, et al. Synthesis of the literature on the effectiveness of regional anesthesia for cataract surgery. Ophthalmology 2003 (in press).

3 Au Eong KG, Lim TH, Lee HM, et al. Subjective visual experience during phacoemulsification and intraocular lens implantation using retrobulbar anesthesia. J Cataract Refract Surg 2000;26:842-6.

4 Torrance GW. Measurement of health state utilities for economic appraisal: a review. J Health Econ 1985;5:1-30.

5 Torrance GW. Social preferences for health states: an empirical evaluation of three measurement techniques. Socioeconomic Planning Sciences $1976 ; 10: 128-36$. 